\title{
Energy-Spectral Efficiency Trade-Off in Virtual MIMO Cellular Systems
}

\author{
Xuemin Hong, Member, IEEE, Yu Jie, Cheng-Xiang Wang, Senior Member, IEEE, \\ Jianghong Shi, and Xiaohu Ge, Senior Member, IEEE
}

\begin{abstract}
Virtual multiple-input multiple-output (V-MIMO) technology promises significant performance enhancements to cellular systems in terms of spectral efficiency (SE) and energy efficiency (EE). How these two conflicting metrics scale up in large cellular V-MIMO networks is unclear. This paper studies the EE-SE trade-off of the uplink of a multi-user cellular V-MIMO system with decode-and-forward type protocols. We first express the trade-off in an implicit function and further derive closed-form formulas of the trade-off in low and high SE regimes. Unlike conventional MIMO systems, the EE-SE trade-off of the V-MIMO system is shown to be susceptible to many factors including protocol design (e.g., resource allocation) and scenario characteristics (e.g., user density). Focusing on the medium and high $\mathrm{SE}$ regimes, we propose a heuristic resource allocation algorithm to optimize the EE-SE tradeoff. The fundamental performance limits of the optimized VMIMO system are subsequently investigated and compared with conventional MIMO systems in different scenarios. Numerical results reveal a surprisingly chaotic behavior of V-MIMO systems when the user density scales up. Our analysis indicates that low frequency reuse factor, adaptive resource allocation, and user density control are critical to harness the full benefits of cellular V-MIMO systems.
\end{abstract}

Index Terms-Energy efficiency, spectral efficiency, virtual MIMO, adaptive resource allocation.

\section{INTRODUCTION}

$\mathbf{V}$ IRTUAL multiple-input multiple-output (V-MIMO) technology enables distributed wireless devices to form virtual antenna arrays (VAAs) and achieve performance gains comparable to conventional MIMO systems [1]-[7]. The application of V-MIMO is particularly promising in cellular networks and sensor/ad-hoc networks, where the devices are equipped with a small number of antennas due to size constraints. In cellular networks, it is commonly assumed

Manuscript received October 10, 2012; revised March 20, 2013. The authors acknowledge the support from the National Natural Science Foundation of China (NSFC) (Grant No.: 61201195, 61210002, and 61271224), the Natural Science Foundation of Fujian (2012J01292), the Opening Project of the Key Laboratory of Cognitive Radio and Information Processing (Guilin University of Electronic Technology), Ministry of Education (No.: 2013KF01), the Ministry of Science and Technology (MOST), China, the International Science and Technology Collaboration Program (Grant No.: 0903), and the Hubei Provincial Science and Technology Department (Grant No.: 2011BFA004).

X. Hong, Y. Jie, and J. Shi are with the School of Information Science and Technology, Xiamen University, Xiamen 361005, Fujian, China (email: $\{$ xuemin.hong, jieyu, shijh\}@xmu.edu.cn).

C.-X. Wang is with the Joint Research Institute for Signal and Image Processing, School of Engineering and Physical Sciences, Heriot-Watt University, Edinburgh, EH14 4AS, UK (e-mail: cheng-xiang.wang@hw.ac.uk).

$\mathrm{X} . \mathrm{Ge}$ is with the Department of Electronics and Information Engineering, Huazhong University of Science and Technology, Wuhan 430074, Hubei, China (e-mail: xhge@mail.hust.edu.cn).

Digital Object Identifier 10.1109/JSAC.2013.131013. that base stations (BSs) are equipped with multiple antennas and therefore, a V-MIMO link involves only one VAA (e.g., [1]-[3], [8]-[12]). In sensor/ad hoc networks, there is no infrastructure and hence VAAs are usually deployed in both ends of a V-MIMO link (e.g., [13]-[17]).

The essence of V-MIMO lies in the integration of MIMO and relay technologies. As a result, V-MIMO systems naturally inherit a large design space incorporating various combinations of MIMO and relay schemes. A wealth of VMIMO designs have been proposed in the literature. On one hand, from the MIMO perspective, V-MIMO systems can be designed to achieve diversity gains [13], [16], [18]-[21], multiplexing gains [14], [15], and/or beamforming gains [22], [23] using various distributed coding and signal processing techniques. Moreover, the MIMO signaling can be designed to treat cooperative relays in a VAA in a non-selective [1], [2], [8], [13], [20] or selective [9], [10], [14] fashion. On the other hand, from the relay perspective, V-MIMO systems can adopt either amplify-and-forward (AF) [8], [9], [22]-[24], decode-and-forward (DF) [2], [3], [18], [25], compress-andforward (CF) [26], [27], or hybrid [11] schemes to complete a transmission in two-hops [1], [8], [9] or multi-hops [2], [3], [14], [18], [20], [25], [28]. Furthermore, the source can transmit information to the relays in a broadcast [16], [18] or multicast fashion [2], [3], [8], [9].

Parallel to the proliferation of V-MIMO designs, the existing literature has adopted various frameworks to evaluate the performance of V-MIMO systems. An evaluation framework mainly includes three aspects: network scale, network topology, and performance metric. In terms of network scale, VMIMO systems can be studied at either the link level or system level. Link level studies [1], [20], [24] concern only one VMIMO link, while system level studies [8], [9], [17] consider multiple V-MIMO links. System level studies should take into account additional factors such as the spatial distribution of users, multi-user interference, frequency reuse, and channel path loss. In terms of network topology, a fixed topology [10], [11], [13]-[16] or random topology [8], [9], [17] can be applied. The former assumes fixed VAA topologies, while the latter takes into account the randomness of the connectivity between nodes forming a VAA. In addition to different network models, different performance metrics can be applied. Popular metrics include ergodic capacity [1]-[3], [8], [9], [27], outage capacity [10], [28], bit error rate (BER) [14], [20], [25], [27], delay [15], and energy-efficiency [13], [18], [25], [29].

Due to the large design space of V-MIMO, in this paper we restrict our analysis to a typical V-MIMO system that adopts 
DF, two-hop, and broadcast based protocols. To our consideration, such a V-MIMO system represents the most practical case to be deployed in cellular networks. First, compared with AF, DF eliminates the error propagation problem [8], [9] and works better with high user densities and high levels of interference. DF also shows a significantly lower complexity than $\mathrm{CF}$ and hence, it is more suitable for mobile terminals. Second, two-hop and broadcast-based protocols have small signaling overhead compared with multi-hop and multicastbased protocols [2], [3]. Therefore, they are more suitable for cellular systems.

This paper introduces a novel performance evaluation framework and applies it to reveal new insights of large scale cellular V-MIMO systems. Our framework is featured with a system-level stochastic network model and a new performance metric. Previous works on V-MIMO are mostly constrained to fixed network topologies. To this regard, there only exists a few articles that take into account the VAA connectivity aspects with random network topologies [8], [9], [17]. Among these works, only [9] has studied V-MIMO in the context of cellular systems with an AF, multi-cast based protocol. In this paper, we complement the work of [9] to study a different V-MIMO system based on DF and broadcast-based protocols.

Another important feature of our framework is the use of a new performance metric: the energy-efficiency (EE) spectralefficiency (SE) trade-off. Driven by practical demands of faster and greener mobile communications, the design of cellular systems is challenged by effective joint optimization of both the SE and EE. SE indicates how efficiently the system bandwidth is utilized, while EE measures how efficiently the energy is consumed. Although optimizing either EE or SE can be seen as matured fields, the joint optimization of both metrics is less-explored and has emerged as a new frontier for wireless communications research in recent years. It is well-known that maximizing EE and SE are conflicting objectives and there exists a fundamental trade-off between EE and SE. This tradeoff sets theoretical limits on the optimization performance and has inspired numerous research. The EE-SE trade-off in the low $\mathrm{SE}$ regime has been studied for single-input single-output [30], [31], MIMO [32], [33], multi-user [34], multi-hop [35], and cooperative communication systems [36]-[39]. Moreover, the trade-off in the high SE regime has been studied for multiaccess [31], [39], [40], MIMO [33], [41], and MIMO broadcast systems [42]. To our best knowledge, the EE-SE trade-off of large scale V-MIMO systems has never been studied.

Our contributions in this paper are summarized as follows:

1) We establish a general theoretical framework to evaluate the EE-SE trade-off of large-scale V-MIMO systems using DF type protocols. Our framework takes into account the spatial structure of a network and is essentially different from existing works. This framework can be easily extended to other types of large-scale V-MIMO systems and cooperative communication systems.

2) We derive closed-form approximations of the EE-SE trade-off for the V-MIMO system in both the high SE regime and low SE regime. To this end, we propose a new approximation formula for MIMO capacity in the high SE regime. This formula decouples the influence of signal-to-noise ratio (SNR) and antenna numbers.
Therefore it has wider applications in the analytical evaluation of other types of MIMO systems involving random numbers of antennas.

3) We apply the EE-SE trade-off concept to protocol design and formulate a general problem of joint EESE optimization. A heuristic power and time allocation algorithm is proposed to solve the optimization problem.

4) We discover the surprisingly chaotic behavior of VMIMO when the density of VAA varies. Moreover, we reveal the fundamental importance of frequency reuse. Our discussions argue that at the system level, the design of V-MIMO should pay attention to several new aspects that have been largely neglected in the V-MIMO literature.

The remainder of this paper is organized as follows. Section II describes the system model. A general characterization of the EE-SE trade-off is given in Section III. Section IV derives the closed-form formulas of the EE-SE trade-off in asymptotic cases. Section $\mathrm{V}$ proposes a heuristic algorithm to optimize the EE-SE trade-off, followed by numerical results and discussions in Section VI. Finally, conclusions are drawn in Section VII.

\section{SySTEM MODEL}

We consider the uplink of a large cellular V-MIMO network with multiple BSs and mobile users. A fraction of the users are active users (sources) that have messages to transmit to the BS. Other users are cooperative users (relays) that are willing to form VAAs with active users to facilitate V-MIMO transmission.

Before the transmission of effective data can start, the following initialization procedure is assumed. (1) An active user broadcasts messages locally to probe nearby inactive users; (2) Inactive users who can successfully decode the message become cooperative users and form a VAA with the active user. (3) The active user reports to the BS about its service demand and VAA size. (4) The BS conducts centralized scheduling and sends configuration parameters (e.g., transmit power, channel allocation, time fraction allocation, and MIMO mode, etc.) to each VAA. Ideally, the above procedure should be repeated as least once within the channel coherence time. This leads to increased signaling overhead. However, compared with conventional cellular systems, only the first two steps cause additional overheads. Because the first two steps can be performed in a local, distributed, and concurrent fashion, we expect the overall overhead to be acceptable and should scale well with the density of VAAs.

A V-MIMO transmission consists of two phases: local broadcasting (Phase I) and distributed MIMO access (Phase II). We assume that Phase I and Phase II operate in a timedivision fashion. The fraction of time allocated to Phase II is denoted as $\varepsilon \in(0,1)$, which is an adjustable parameter controlled by BSs. Each BS is equipped with $r$ antennas while each mobile user has only one antenna. All wireless channels are subject to Rayleigh fading and path loss with a constant path loss exponent $\alpha=4$. The bandwidth of the entire cellular system is $W$.

Based on the above system description, our research objective is to gain theoretical insights into the impacts of network 
scale and resource allocation on the EE-SE trade-off of VMIMO systems. Furthermore, based on such insights, we aim to explore how resource allocation can be optimized with respect to the EE-SE trade-off.

\section{A. Stochastic model of large-scale V-MIMO networks}

The performance of V-MIMO depends largely on the system's random spatial layout, the essence of which can be captured by stochastic geometry models [43]. The BSs, mobile users, and active users are assumed to be spatially distributed in an Euclidean plane according to some homogeneous Poisson point processes (PPPs) denoted as $\Phi_{b}, \Phi_{u}$, and $\Phi_{v}$, respectively. Users become active users randomly and each active user corresponds to a VAA. The densities of $\Phi_{b}, \Phi_{u}$, and $\Phi_{v}$ are denoted by $\lambda_{b}, \lambda_{u}$, and $\lambda_{v}$, respectively, where $\lambda_{b} \leq \lambda_{v} \leq \lambda_{u}$. We note that although the traditional cellular model places BSs on regular lattices, in reality, the locations of BSs are often randomized due to site availability and local coverage considerations (e.g., cover hot spots). A recent study revealed that the PPP model, while being much more tractable, is no less accurate than the traditional regular lattice model [44].

We assume that an active user only communicates with the nearest BS. The BS coverage areas can therefore be described by a Voronoi tessellation defined on $\Phi_{b}$. The coverage area of a BS is called a BS cell. Similarly, we assume that each inactive user can only cooperate with the nearest active user. Therefore, each active user defines a VAA area, within which the inactive users can participate in the local VAA initiated by the active user. The VAA areas are described by another Voronoi tessellation defined on $\Phi_{v}$. The VAA area of an active user is also called a VAA cell. Fig. 1 shows a snapshot of the random spatial layout of our system model. The solid (red) and dashed (blue) lines show the boundaries of BS cells and VAA cells, respectively. In reality, the boundaries of BS and VAA cells are blurred due to channel shadowing and fading. Nevertheless, in our subsequent analysis, we are mainly interested in the cell size rather than the exact shape of the cell. As far as the cell sizes (cell areas) are concerned, the random effects of channels tend to balance out, and the Voronoi model serves as a good theoretical model to capture the essence of cell size variation.

\section{B. Phase I: Local broadcasting}

In the first phase, each active user broadcasts its own message with identical power $P$. Using adaptive modulation and coding techniques, the broadcast message is designed to be received at a signal-to-interference-plus-noise ratio (SINR) above $\chi$. An inactive user becomes a participant of the VAA only when it can successfully decode the message from the nearest active user. The number of antennas (users) in a VAA is a random variable denoted by $t$. In the worst case, no inactive user can decode the broadcasted message and the VAA includes only the active user. To achieve high broadcast capacity, we assume that the entire system bandwidth $W$ is spatially reused by all VAAs,i.e., all active users broadcast with full bandwidth $W$ to achieve a spatial frequency reuse

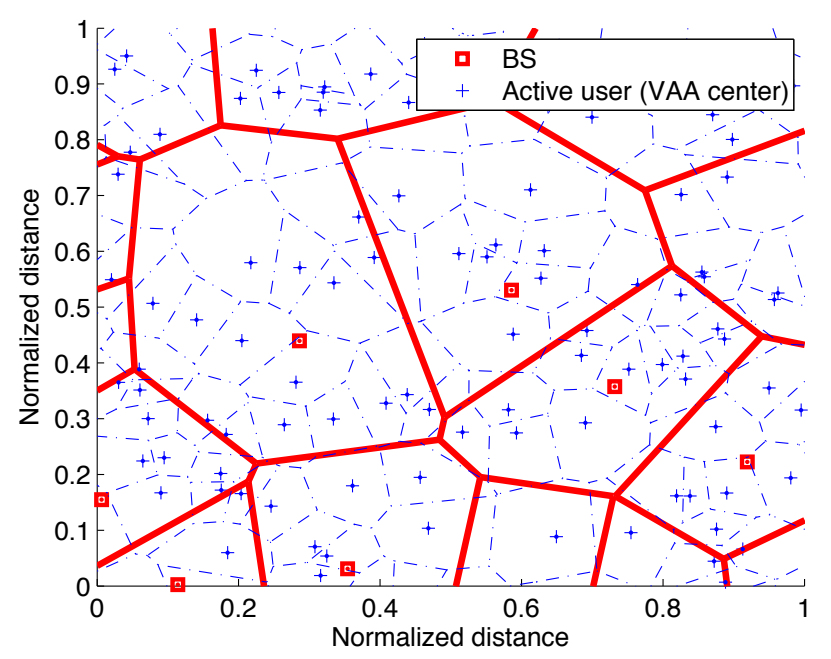

Fig. 1. Spatial structure of a cellular V-MIMO system with Voronoi BS cells (solid line) and Voronoi VAA cells (dashed line).

factor of 1. Consider an arbitrary link from an active user to an inactive user, the receive SINR is given by

$$
\gamma=\frac{P h}{P I+W N_{0}}=\frac{h}{I+\rho^{-1}}
$$

where $P$ is the transmit power, $I$ is the accumulated interference normalized to $P, \rho$ is normalized transmit power (or transmit SNR) defined as $\rho=P /\left(W N_{0}\right), N_{0}$ is the power spectral density of Gaussian noise , $h$ is the channel gain given by $h=g d^{-\alpha}, d$ is a random variable (RV) denoting the distance between the active user and the inactive user, and the $\mathrm{RV} g \sim \exp (1)$ follows an exponential distribution with unit mean to represent the power gain of Rayleigh fading channels. The accumulated interference $I$ is given by

$$
I=\sum_{i} g_{i} d_{i}^{-\alpha}, \quad(i=1,2, \ldots \ldots \infty)
$$

where $i$ is the index of interfering active users, $d_{i}$ is the distance from the inactive user to the $i$ th interferer, $g_{i} \sim \exp (1)$ are RVs to account for Rayleigh fading in the interference channels. According to the spatial PPP model, the probability density function (PDF) of $d$ is given by [43]

$$
f_{d}^{\prime}(x)=2 x \pi \lambda_{v} e^{-\pi \lambda_{v} x^{2}}, \quad x \in(0, \infty)
$$

In the context of wireless networks, the above PDF has a drawback. When $d \in(0,1)$, we have $d^{-\alpha}>1$, meaning that the receive power becomes greater than the total transmit power, which is unrealistic. Following a common practice to eliminate this drawback, in this paper we set $d \in[1, \infty)$ and adopt a slightly modified PDF given by

$$
f_{d}(x)=2 x \pi \lambda_{v} e^{\pi \lambda_{v}\left(1-x^{2}\right)}, \quad x \in(1, \infty)
$$

Numerical results show that the difference between (3) and (4) become significant in high user density scenarios when $\lambda_{v}>0.1$. 


\section{Phase II: Distributed MIMO access}

The second phase of V-MIMO transmission is distributed MIMO access, where each VAA transmits the message received in Phase I to the associated BS. Consider an arbitrary transmission link from a VAA to a $\mathrm{BS}$, the receive SINR is given by

$$
\tilde{\chi}=\frac{\tilde{P} \tilde{h}}{\tilde{W}\left(N_{0}+N_{I}\right)}
$$

where $\tilde{W}$ is the bandwidth allocated to the VAA, $\tilde{P}$ is the total transmit power of the VAA, $\tilde{h}=\tilde{d}^{-\alpha}$ is the path loss of the channel, $\tilde{d}$ is the distance from the VAA to $\mathrm{BS}, N_{0}$ is the power spectral density of white Gaussian noise, and $N_{I}$ is the power spectrum density of inter-cell interference, which is also assumed to be a white Gaussian noise. In (5), we only consider path loss in the channel because Rayleigh fading will be naturally accounted for in the calculation of MIMO capacity. Similar to (4), the PDF of $\tilde{d}$ follows

$$
f_{\tilde{d}}(x)=2 x \pi \lambda_{b} e^{\pi \lambda_{b}\left(1-x^{2}\right)}, \quad x \in[1, \infty) .
$$

In (5), although a numerical characterization of $N_{I}$ is possible [44], it leads to intractable results without revealing any theoretical insights. To facilitate our analysis, we will subsequently neglect the inter-cell interference. It is worth noting that the inter-cell interference $N_{I}$ is universal to both conventional and V-MIMO cellular systems. In other words, inter-cell interference degrades the performance of conventional and VMIMO cellular systems to a similar extent. Therefore, this simplifying assumption does not compromise our goal of revealing the relative pros and cons of V-MIMO.

\section{Rate balance assumption}

A key assumption to our system model is the "rate balance" assumption. This assumption states that, over a long term, the average data rate broadcasted in Phase I should equal the average data rate successfully received by the BS in Phase II. This assumption guarantees that no information is lost in the relay process and no unnecessary energy is spent. Therefore, the system operates in an optimal state in terms of EE and SE trade-off. In practice, rate balance can be achieved via jointscheduling of Phase I and Phase II. It can be enforced in each round of V-MIMO transmission. For example, the VAA can apply power control in Phase II to transmit all the information received in Phase I to the BS. Alternatively, rate balance can be achieved in an average sense over a long period. For example, the VAA can buffer the data received in Phase I and transmit to the BS with varying data rates according to channel conditions. The former case using power control is assumed in this paper.

To better explain this assumption, let's consider a simple case of a single V-MIMO link. Denote the average transmission rate from an active user (source) to cooperative users (relays) in Phase I as $\bar{C}$, the average rate between a VAA and the BS in Phase II as $\tilde{\tilde{C}}$, the energy consumed in Phase I as $U$, and the energy consumed in Phase II as $\tilde{U}$. The average energy-per-bit can be evaluated as $E_{b}=(U+\tilde{U}) / \min (\bar{C}, \tilde{C})$. Because $U$ and $\tilde{U}$ are monotonically increasing functions of $\bar{C}$ and $\overline{\tilde{C}}$, respectively, it is easy to prove that given a targeted rate (i.e., SE) $C_{0}$, the minimum $E_{b}$ is achieved when $\bar{C}=\overline{\tilde{C}}=C_{0}$.
Hence the best EE-SE trade-off is obtained with equal average rates of the two phases. Here, the best EE-SE trade-off means that the highest $\mathrm{EE}$ (i.e., lowest $E_{b}$ ) is achieved at all SE values given the set of system parameters.

The maximum transmission rate of a VAA in Phase I is

$$
R=W \log _{2}(1+\chi)
$$

where $\chi$ is the SINR threshold above which the broadcasted message can be successfully decoded by cooperative users. The maximum transmission rate of a VAA in Phase II is

$$
\tilde{R}=\tilde{W} f_{\mathrm{MIMO}}(t, r, \tilde{\chi})=\tilde{W} \mathbb{E}_{\mathbf{H}}\left(\log _{2}\left|\mathbf{I}_{r}+\frac{\tilde{\chi}}{t} \mathbf{H H}^{\dagger}\right|\right)
$$

where $f_{\text {MIMO }}(t, r, \tilde{\chi})$ denotes the capacity of $t \times r$ i.i.d. Rayleigh faded MIMO channels at a SINR of $\tilde{\chi}, \mathbb{E}(\cdot)$ denotes expectation and $(\cdot)^{\dagger}$ denotes the conjugate transpose of a matrix. We note that an idealized MIMO channel is assumed here for analytical tractability . In practice, MIMO capacity could be degraded due to channel correlation resulted from insufficient antenna spacing [45]. From the rate balance assumption we have

$$
\mathbb{E}[(1-\varepsilon) R]=\mathbb{E}_{t, \tilde{W}}[\varepsilon \tilde{R}]
$$

where $\varepsilon$ is the time fraction allocated to Phase II.

In (9), the right-side expectation is taken over RVs $t$ and $\tilde{W}$. The bandwidth $\tilde{W}$ of a VAA depends on both the frequency reuse schemes over the entire network and BS bandwidth allocation schemes within a single cell. This opens a wealth of possibilities for a detailed characterization of $\tilde{W}$. For a general discussion, we assume an ideal frequency reuse and bandwidth allocation scheme, which together gives $\mathbb{E}(\tilde{W})=K_{w} W \lambda_{b} / \lambda_{v}$, where $K_{w}$ is a constant parameter. This assumption is based on two intuitive principles. First, the overall bandwidth resources should increase linearly with the density of BSs (i.e., spatial reuse gains). Second, consider perfect fairness among all VAAs, the average bandwidth available to each VAA should decrease linearly with the density of VAAs. This simplifying assumption can capture the essence of all frequency reuse and bandwidth allocation schemes to facilitate our subsequent analysis. To further validate this assumption, we test a special case where the frequency reuse factor is 1 and the system bandwidth $W$ is equally divided among all VAAs within a cell. The number of VAAs in a cell is a discrete RV, whose probability mass function (PMF) can be evaluated numerically [46]. Numerical results reveal that for a practical range of $\lambda_{v} / \lambda_{b} \in[5,200]$, we have $K_{w} \approx 1.43$. Because the exact value of $K_{w}$ is trivial to our following discussions, we will subsequently set $K_{w}=1$ for simplicity. It follows that

$$
\mathbb{E}(\tilde{R})=\frac{\lambda_{b}}{\lambda_{v}} W \mathbb{E}_{t}\left[f_{\mathrm{MIMO}}(t, r, \tilde{\chi})\right]
$$

Substituting (7) and (10) into (9) we get

$$
(1-\varepsilon) \mathbb{E}_{\chi}\left[\log _{2}(1+\chi)\right]=\varepsilon \frac{\lambda_{b}}{\lambda_{v}} \mathbb{E}_{t}\left[f_{\mathrm{MIMO}}(t, r, \tilde{\chi})\right] .
$$

This equation implies adaptive power control in Phase II to enforced rate balance. Consider strict fairness so that all VAAs broadcast at the same rate in Phase I (i.e., the same 


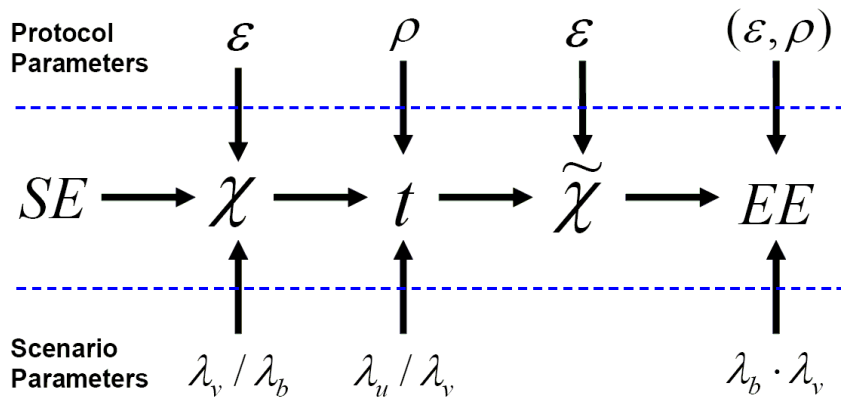

Fig. 2. Parameters related to the EE-SE trade-off of V-MIMO systems.

SINR threshold $\chi$ is used by all VAAs), it follows that $\mathbb{E}_{\chi}\left[\log _{2}(1+\chi)\right]=\log _{2}(1+\chi)$.

So far, we have introduced our system model, which is characterized by best-effort broadcasting during VAA establishment, adaptive power control during MIMO access, decode-and-forward relay, and maximum frequency reuse.

\section{EE-SE TRADE-OFF}

Assuming that a system achieves its maximum capacity, the SE $S$ is directly related to the channel capacity and is given by $S \leq C / W$, where $C$ is the capacity of the system and $W$ is the system bandwidth. In the context of the V-MIMO system studied in our paper, the average spectrum efficiency $S$ of a VAA can be evaluated as

$$
S=(1-\varepsilon) \frac{\lambda_{v}}{\lambda_{b}} \log _{2}(1+\chi)=\varepsilon \mathbb{E}_{t}\left[f_{\mathrm{MIMO}}(t, r, \tilde{\chi})\right] .
$$

On the other hand, various EE metrics have been proposed in the literature, including the energy-per-bit normalized to noise power spectral density ratio $\left(E_{b} / N_{0}\right)$, the bit-per-Joule capacity, the rate per energy, and the Joule-per-bit. All these EE metrics are essentially the same. In this paper we choose the widely used $E_{b} / N_{0}$ as our EE metric. Although both radiated power and circuit power can be considered in calculating the EE [33], in this paper we consider only the radiated power to focus on the fundamental limits. The objective of EE-SE trade-off study is to express EE as a function of SE, or express $\mathrm{SE}$ as a function of EE. In this paper we choose the former approach.

In Fig. 2, we illustrate the relationships among various parameters to show how the EE and SE are inherently related in the V-MIMO system. For better conceptual understanding, we distinguish two types of parameters: protocol parameters and scenario parameters. Protocol parameters, including the normalized Phase I transmit power $\rho$ and time allocation parameter $\varepsilon$, are adaptable factors that can be adjusted by protocol design. Scenario parameters, including $\lambda_{b}, \lambda_{u}, \lambda_{v}$, and $r$, are unadjustable factors defined by a particular communication scenario. As shown in Fig. 2, it takes four steps to calculate EE from SE. The first step can be easily derived from (12) as

$$
\chi=2^{\frac{S \lambda_{b}}{(1-\varepsilon) \lambda_{v}}}-1
$$

The rest three steps are more complicated and will be explained separately in the following three subsections.

\section{A. PDF of VAA antenna numbers $t$}

In our system model, each inactive user is assumed to be associated with the nearest active user. Let $M^{\prime}$ denote the number of associated inactive user per active user, the PMF of $M^{\prime}$ is given by [46]

$$
f_{M^{\prime}}(n)=\frac{3.5^{3.5}}{\Gamma(3.5) n !} \frac{\Gamma(n+3.5)\left(\frac{\lambda_{w}}{\lambda_{v}}\right)^{n}}{\left(\frac{\lambda_{w}}{\lambda_{v}}+3.5\right)^{n+3.5}}
$$

where $\Gamma(\cdot)$ denotes the Gamma function, $\lambda_{w}=\lambda_{u}-\lambda_{v}$ denotes the density of inactive users, and $(\cdot)$ ! denotes factorial. Consider a typical inactive user with an arbitrary location on the plane, the received SINR is a random variable. Following a similar procedure in [44] but applying the modified PDF of $r$ given by (4), the complementary CDF of SINR in Phase I can be derived as

$$
\begin{aligned}
F_{\gamma}(x) & =\pi \lambda_{v} e^{\pi \lambda_{v}} \int_{1}^{\infty} e^{-a y-b y^{2}} \mathrm{~d} y \\
& =\frac{\pi^{3 / 2} \lambda_{v} e^{\lambda_{v} \pi}}{\sqrt{b}} e^{\frac{a^{2}}{4 b}} \mathrm{Q}\left(\sqrt{2 b}+\frac{a}{\sqrt{2 b}}\right)
\end{aligned}
$$

where $a=\lambda_{v} \pi\left[1+\sqrt{x}\left(\frac{\pi}{2}-\arctan \left(\frac{1}{\sqrt{x}}\right)\right]\right.$ and $b=x \rho^{-1}$. According to the DF relay protocol, an inactive user can become a cooperative user (i.e., participant of a VAA) only if it can receive Phase I broadcasting with a SINR higher than $\chi$. The probability for an inactive user to become a cooperative user can now be calculated as $F_{\gamma}(\chi)$. Let $M$ denote the number of cooperating users per active user, we have $M \sim \mathrm{B}\left(M^{\prime}, F_{\gamma}(\chi)\right)$, i.e., $M$ follows a binomial distribution with parameters $M^{\prime}$ and $F_{\gamma}(\chi)$. Since $M^{\prime}$ is a random variable, the PMF of $M$ can be obtained by taking the expectation over $M^{\prime}$, i.e.,

$$
f_{M}(n)=\sum_{m=0}^{\infty} f_{M}^{\prime}(n) \mathrm{C}_{m}^{n} F_{\gamma}(\chi)^{n}\left[1-F_{\gamma}(\chi)\right]^{m-1} \quad(n \geq 0)
$$

where $\mathrm{C}_{m}^{n}=m ! / n$ !. Finally, since a VAA includes not only cooperating users but also the active user, the PMF of the random antenna numbers $t$ in a VAA is given by $f_{t}(n)=$ $f_{M}(n-1)(n \geq 1)$.

\section{B. Phase II SINR $\tilde{\chi}$}

Given SE $S$, VAA antenna PDF $f_{t}(n)$, Phase II time fraction $\varepsilon$, and receive antenna number $r$, the value of $\tilde{\chi}$ is uniquely determined by (12). Apart from some special cases, it is difficult to simplify the expectation in (12) and express $\tilde{\chi}$ as an explicit function of $S$. However, because $f_{\text {MIMO }}(t, r, \tilde{\chi})$ is a monotonously increasing function of $\tilde{\chi}$, highly efficient numerical methods such as the Newton's method can be used to quickly compute $\tilde{\chi}$ as a function of $S$. To facilitate such numerical computations, an accurate approximation of the MIMO capacity can be used in (8) as [47]

$$
\begin{aligned}
& f_{\mathrm{MIMO}}(t, r, \tilde{\chi}) \approx-\frac{t}{\ln (2)}\left[-(1+\beta) \ln (\sqrt{\tilde{\chi}})+q_{0}(\tilde{\chi}) r_{0}(\tilde{\chi})\right. \\
& \left.+\ln \left(r_{0}(\tilde{\chi})\right)+\beta \ln \left(q_{0}(\tilde{\chi}) / \beta\right)\right] .
\end{aligned}
$$


In (18), we have $\beta=r / t$,

$$
\begin{aligned}
& q_{0}(\tilde{\chi})=\frac{-1-u(\tilde{\chi})+v(\tilde{\chi})}{2 \sqrt{\tilde{\chi}}} \\
& r_{0}(\tilde{\chi})=\frac{-1+u(\tilde{\chi})+v(\tilde{\chi})}{2 \sqrt{\tilde{\chi}}}
\end{aligned}
$$

where $u(\tilde{\chi})=\tilde{\chi}(1-\beta)$ and $v(\tilde{\chi})=$ $\sqrt{1+2 \tilde{\chi}(1+\beta)+\tilde{\chi}^{2}(1-\beta)^{2}}$. This accurate approximation has been derived by assuming a large number of $t$ and $r$. However, its accuracy has been deemed acceptable even for a small number of antennas [47]. Our numerical tests show that the approximation error is lower than $1 \%$ given $r \geq 4$.

\section{Energy efficiency}

The average energy efficiency can be measured in terms of $E_{b} / N_{0}$ and is given by

$$
\frac{E_{b}}{N_{0}}=\frac{(1-\varepsilon) Q+\varepsilon \tilde{Q}}{S \mathbb{E}[\tilde{W}] N_{0}}=\frac{1}{S} \frac{\lambda_{v}}{\lambda_{b}}\left(\frac{(1-\varepsilon) Q+\varepsilon \tilde{Q}}{W N_{0}}\right)
$$

where $Q=\mathbb{E}[P]=P$ is the average power consumption in Phase $\mathrm{I}$ and $\tilde{Q}=\mathbb{E}[\tilde{P}]$ is the average power consumption in Phase II. From (5) we have

$$
\tilde{Q}=\mathbb{E}[\tilde{P}]=\mathbb{E}[\tilde{\chi}] \mathbb{E}[\tilde{W}] \mathbb{E}\left[\tilde{h}^{-1}\right]=\tilde{\chi} \frac{\lambda_{b}}{\lambda_{v}} W N_{0} \mathbb{E}\left[\tilde{h}^{-1}\right]
$$

where $\tilde{h}$ is the random channel path loss between the VAA and the BS. Based on the system model we get

$$
\begin{aligned}
\mathbb{E}\left[\tilde{h}^{-1}\right] & =e^{\lambda_{b} \pi}\left(\lambda_{b} \pi\right)^{-\frac{\alpha}{2}} \Gamma\left[(\alpha / 2+1),\left(\lambda_{b} \pi\right)^{\frac{\alpha}{2}}\right] \\
& \approx\left(\lambda_{b} \pi\right)^{-\frac{\alpha}{2}} \Gamma(\alpha / 2+1) \quad\left(\lambda_{b} \ll 1\right) .
\end{aligned}
$$

Substituting (22) and (24) into (21), we get

$$
\frac{E_{b}}{N_{0}}=\frac{\lambda_{v}}{\lambda_{b} S}\left[(1-\varepsilon) \rho+\frac{2 \tilde{\chi} \varepsilon}{\pi^{2} \lambda_{v} \lambda_{b}}\right]
$$

where $\rho=P /\left(W N_{0}\right)=Q /\left(W N_{0}\right)$. For comparisons with conventional cellular systems, it is more convenient to consider the $E_{b} / N_{0}$ normalized to the channel gains $\mathbb{E}\left[\tilde{h}^{-1}\right]$. The normalized $E_{b} / N_{0}$, denoted as $E$, is given by

$$
E=\frac{1}{S}\left[\frac{\pi^{2}}{2}(1-\varepsilon) \rho \lambda_{v} \lambda_{b}+\tilde{\chi} \varepsilon\right]
$$

where $\tilde{\chi}$ is the Phase II SNR and can be numerically calculated as a function of $S$. So far, we have established the theoretical framework to numerically evaluate the EE-SE trade-off of the V-MIMO system.

\section{Asymptotic Analysis of the EE-SE Trade-OFF}

Although it is difficult to find a general closed-form expression for the EE-SE trade-off, in this Section we will show that the EE-SE trade-off can be well-approximated by closed-form formulas when $S \rightarrow \infty$ and $S \rightarrow 0$, i.e., in the high SE regime and low SE regime.

\section{A. High SE regime}

In the four steps of computing $\mathrm{EE}$ from SE, only the third step involves numerical computation of $\tilde{\chi}$. A close look reveals that the difficulty of obtaining an analytical expression for $\tilde{\chi}$ comes from the fact that $\tilde{\chi}$ and VAA antenna number $t$ cannot be separated in the general MIMO capacity formula given by (18). Motivated by the fact that an accurate and invertible formula exists to calculate the MIMO capacity in the case of symmetrical antennas (i.e., $t=r$ ) [33], [48], we can rewrite (18) as

$$
f_{\mathrm{MIMO}}(t, r, \tilde{\chi})=\min (t, r) \mathrm{G}(\tilde{\chi})+\Delta(t, r, \tilde{\chi})
$$

where the function $\mathrm{G}(x)$ is defined as [48]

$$
\mathrm{G}(x)=2 \log _{2}\left[1+x-\frac{1}{4} \mathrm{~L}(x)\right]-\frac{1}{\ln (2) 4 x} \mathrm{~L}(x)
$$

with $\mathrm{L}(x)=(\sqrt{4 x+1}-1)^{2}$.

In the right hand side of (27), the first item is the capacity in the symmetrical antenna case and the second item is the capacity difference. Function $\mathrm{G}(x)$ has a desirable property as being analytically invertible [33], i.e.,

$$
\mathrm{G}^{-1}(x)=-\frac{1}{4}+\frac{1}{4}\left\{1+\left[\mathrm{W}_{0}\left(-2^{-\left(\frac{x}{2}+1\right)} e^{-\frac{1}{2}}\right)\right]^{-1}\right\}^{2}
$$

where $\mathrm{W}_{0}(x)$ denotes the real branch of the Lambert function. The Lambert W function is the inverse function of $f(w)=$ $w e^{w}$ and satisfies $\mathrm{W}(z) e^{\mathrm{W}(z)}=z$ with complex values of $w$ and $z$. Its real branch $\mathrm{W}_{0}$ maps inputs from interval $\left[-e^{-1},+\infty\right)$ to interval $[-1,+\infty)$ and is a monotonically increasing function.

It is well known that in the high SNR regime, the MIMO capacity scales linearly with the number of antenna pairs. This suggests that $\Delta(t, r, \tilde{\chi})$ should approach a constant limit $\Delta^{*}(t, r)$ when SNR $\tilde{\chi}$ tends to infinity. Numerical results based on (18) confirm that when $\tilde{\chi} \geq 40 \mathrm{~dB}, \Delta(t, r, \tilde{\chi})$ can be well approximated by $\Delta(t, r, \infty)=\Delta^{*}(t, r)$ with an approximation error of less than one percent. In Fig. 3, setting $\tilde{\chi}=200 \mathrm{~dB}$, we compute $\Delta^{*}(t, r)$ numerically as a function of $t$ with varying $r$. It is found that $\Delta^{*}(t, r)$ exhibits some interesting properties. Specifically, the curve has a bell shape and we have $\Delta^{*}(t=r / 2, r)=r, \Delta^{*}(t=0, r)=0$, and $\Delta^{*}(t=r, r)=0$. These properties inspire us to approximate $\Delta^{*}(t, r)$ with the following series

$$
\Delta^{*}(t, r) \approx r-\sum_{n=2}^{\infty} K_{n} \frac{2^{n}}{r^{n-1}}\left|t-\frac{r}{2}\right|^{n}, \quad \sum_{n=1}^{\infty} K_{n}=1
$$

where $K_{n}$ are weights that can be optimized numerically. By means of numerical fitting, we are able to get $K_{2}=0.5$, $K_{3}=0.25, K_{4}=0.25$ for a good enough approximation. It follows that

$\Delta^{*}(t, r) \approx r-\frac{2}{r}\left(t-\frac{r}{2}\right)^{2}-\frac{4}{r^{2}}\left|t-\frac{r}{2}\right|^{3}-\frac{8}{r^{3}}\left(t-\frac{r}{2}\right)^{4}(t \leq r)$.

When $t>r$, we have $\Delta^{*}(t, r)=0$. The accuracy of the above approximation is shown in Fig. 3. Through extensive numerical tests we find that the maximum approximation error is lower than $1.3 \%$ for any combinations of $t$ and $r$, while the average approximation error is lower than $0.1 \%$ across all $t \leq r$ for any fixed value of $r$. 


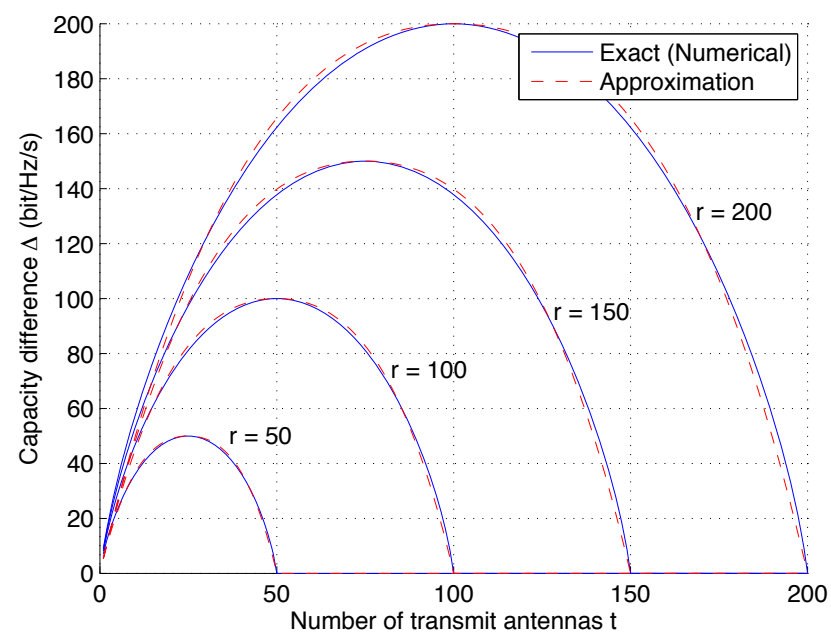

Fig. 3. Numerical results and approximations of the capacity difference $\Delta$ between a general MIMO system and a corresponding symmetrical MIMO system.

Substituting (27) and (31) into (12) we get

$$
S \approx \varepsilon[\bar{N} \mathrm{G}(\tilde{\chi})+\bar{\Delta}]
$$

where $\bar{N}$ is the expectation of $\min (t, r)$ over $t$ given by

$$
\bar{N}=\mathbb{E}_{t}[\min (t, r)]=\sum_{n=1}^{r} n f_{M}(n)+r\left[1-\sum_{n=0}^{n} f_{M}(n)\right]
$$

and $\bar{\Delta}$ is the expectation of $\Delta^{*}(t, r)$ over $t$ given by

$$
\bar{\Delta}=\mathbb{E}_{t}\left[\Delta^{*}(t, r)\right]=\sum_{n=1}^{r} \Delta^{*}(t, r) f_{t}(n) .
$$

From (32), we can write $\tilde{\chi}$ as a function of $S$

$$
\tilde{\chi} \approx \mathrm{G}^{-1}\left(\frac{S-\varepsilon \bar{\Delta}}{2 \varepsilon \bar{t}}\right) .
$$

Substituting (35) into (26), a closed-form approximation formula for the EE-SE trade-off in the high SE regime can be obtained as

$$
E_{\mathrm{high}} \approx \frac{1}{S}\left[\frac{1}{2}(1-\varepsilon) \rho \lambda_{v} \lambda_{b} \pi^{2}+\mathrm{G}^{-1}\left(\frac{S-\varepsilon \bar{\Delta}}{2 \varepsilon \bar{t}}\right) \varepsilon\right] .
$$

Finally, we discuss briefly the gradient of the EE-SE tradeoff curve, where $E_{b} / N_{0}$ is evaluated in the dB scale. It is well know that when $S \rightarrow \infty$, this gradient indicates the spatial degree of freedom (DoF) of a MIMO system [48]. From (36), the gradient of EE-SE trade-off curve in the V-MIMO system can be easily evaluated as

$$
\lim _{S \rightarrow \infty} \frac{d E_{d B}(S)}{d S}=\frac{10 \ln (2)}{\varepsilon \ln (10)}
$$

where $E_{d B}=10 \log _{10}(E)$. For comparison purpose, we note that the gradient of a $t \times r$ MIMO system is $\frac{10 \ln (2)}{\min (t, r) \ln (10)}$.

\section{B. Low SE regime}

The MIMO capacity in the low SNR regime can be approximated by $f_{\mathrm{MIMO}}(t, r, \tilde{\chi}) \approx \sqrt{r \tilde{\chi} / \ln (2)}$ [48], which is independent from the number of transmit antennas $t$. This approximation is deemed accurate for $\tilde{\chi}<-40 \mathrm{~dB}$. It follows that $\tilde{\chi} \approx \varepsilon S^{2} \ln (2) / r$ and the EE-SE trade-off in the low SE regime is given by

$$
E_{\mathrm{low}} \approx \frac{1}{S}\left[\frac{1}{2}(1-\varepsilon) \rho \lambda_{v} \lambda_{b} \pi^{2}+\varepsilon S^{2} \ln (2) / r\right] .
$$

\section{OPTIMIZATION OF PROTOCOL PARAMETERS}

As illustrated in Fig. 2, we have established the EE-SE trade-off function subject to protocol parameters and scenario parameters. From the practical perspective of system design and optimization, it is desirable to understand how the protocol parameters $\rho$ (normalized transmit power of Phase I) and $\varepsilon$ (time fraction of Phase II) can be properly set to give an optimized trade-off curve. Although the importance of resource allocation in communication networks has been widely studied and recognized (e.g., [49], [50]). This topic, however, is still under-explored for large scale V-MIMO systems.

A general formulation of the optimization problem is to maximize the accumulated $\mathrm{EE}$ (i.e., minimize $E_{b} / N_{0}$ ) on a given SE interval $\left[S_{l}, S_{u}\right]$ within which the system operates, i.e.,

$$
\min _{\rho, \varepsilon}\left(\int_{S} E(S) d S\right), \quad S \in\left[S_{l}, S_{u}\right]
$$

A simple case of the above optimization is to maximize $\mathrm{EE}$ at a given SE, which in practice can be the average SE or peak SE of a system.

We note that in reality, $\rho$ is limited by device capability. In (39), we deliberately neglect the constraint on the transmit power $\rho$. This is because for theoretical purpose, we are interested in the optimum solution $\rho^{*}$ that is fundamentally limited by the system itself due to self-interference in Phase I. Such an unbounded optimization problem can be solved because we consider EE as the utility function, so that $\rho$ is inherently constrained.

In general, finding the optimal solution requires an exhaustive search on the 2-dimensional space of $\rho$ and $\varepsilon$. Because $\rho$ has a large dynamic range, this brutal-force approach is rather inefficient. Here we propose a heuristic approach to directly compute a sub-optimal value of $\rho$, denoted as $\rho^{*}$. Given $\rho^{*}$, we can easily search for an optimal $\varepsilon$ as it is bounded in $(0,1)$.

Our heuristic approach targets the high SE regime and is based on the following idea: we want to have as many cooperating users as possible to maximize EE. However, when $\rho$ reaches a threshold, further increasing $\rho$ is not helpful as Phase I broadcasting becomes interference limited. Therefore, we want to have the minimum $\rho$ that can achieve $k$ percent of the best performance given by $\rho \rightarrow \infty$. Recall that in Section III.A, the probability of an inactive user to become a cooperating user is given by $F_{\gamma}(\chi)$. Because this probability is directly related to the average number of cooperating users, it can be used as a convenient performance indicator. To simplify our analysis, we consider the following approximation

$$
F_{\gamma}(x) \approx F_{\gamma}^{*}(x)=\frac{\pi^{\frac{3}{2}} \lambda_{v}}{\sqrt{b}} e^{\frac{a^{2}}{4 b}} \mathrm{Q}\left(\frac{a}{\sqrt{2 b}}\right)
$$


where $a$ is defined in (16) and $b=x \rho^{-1}$. This approximation is deemed valid for $\lambda_{v}<10^{-2}$, which suits most practical scenarios. The 'best performance' can be evaluated as

$$
F_{\gamma}^{\lim }(x)=\lim _{\rho \rightarrow \infty} F_{\gamma}^{*}(x)=\frac{1}{1+\sqrt{x}[\pi / 2-\arctan (1 / \sqrt{x})]} .
$$

The optimization of $\rho$ can now be formalized as

$$
\rho^{*}=\arg \min _{\rho} \frac{F_{\gamma}^{*}(\chi)}{F_{\gamma}^{\lim }(\chi)} \geqslant k, \quad k \in(0,1) .
$$

At the high SE regime, the $Q$ function appeared in (40) can be well approximated by a lower bound given by

$$
\mathrm{Q}(x) \gtrsim \frac{x}{\sqrt{2 \pi}\left(1+x^{2}\right)} e^{-x^{2} / 2} .
$$

Substituting (40), (41) and (43) into (42), we get

$$
\rho^{*} \approx \frac{k}{1-k} \frac{2 \chi}{\left(\pi \lambda_{v}\right)^{2}\left[1+\sqrt{\chi}\left(\frac{\pi}{2}-\arctan (1 / \sqrt{\chi})\right)\right]^{2}}
$$

where $\chi$ can be expressed as a function of SE $S$ according to (13). Equation (44) shows that $\rho^{*}$ can be directly calculated according to a target SE. Given $S \rightarrow \infty$, (44) can be further simplified to

$$
\rho^{*} \approx \frac{k}{1-k} \frac{8}{\pi^{4} \lambda_{v}^{2}}
$$

as a general formula for the high SE regime. Once $\rho^{*}$ is calculated, an exhaustive search on $(0,1)$ can be used to find the optimal value of $\varepsilon$. More details will be provided in the next Section regarding the numerical optimization of $\varepsilon$.

\section{NUMERICAL RESULTS AND DISCUSSIONS}

This section presents numerical results based on our previous analysis and is organized to answer the following two questions: (1) What is the impact of protocol parameters ( $\rho$ and $\varepsilon$ ) on the EE-SE trade-off and how can these parameters be optimized (Figs. 4-8)? (2) What is the impact of scenario characteristics on the optimized EE-SE trade-offs (Figs. 7-11)?

To facilitate our discussion, we define the following four typical scenarios:

- H-H scenario: High user density, high active ratio, $\lambda_{u} / \lambda_{v} / \lambda_{b}=1000: 200: 1$;

- H-L scenario: High user density, low active ratio, $\lambda_{u} / \lambda_{v} / \lambda_{b}=1000: 20: 1$;

- L-H scenario: Low user density, high active ratio, $\lambda_{u} / \lambda_{v} / \lambda_{b}=100: 20: 1$;

- L-L scenario: Low user density, low active ratio, $\lambda_{u} / \lambda_{v} / \lambda_{b}=100: 2: 1$.

The BS density is fixed to one BS per square $\mathrm{km}$, i.e., $\lambda_{b}=$ $10^{-6}$. Furthermore, we distinguish three SE regimes: low SE regime $(S \in[0,0.1]])$, medium $S E$ regime $(S \in(0.1,40])$, and high $\mathrm{SE}$ regime $(S \in(40, \infty))$. Our subsequent discussions will mainly focus on the medium and high SE regimes. Except otherwise mentioned, we set $r=20$.

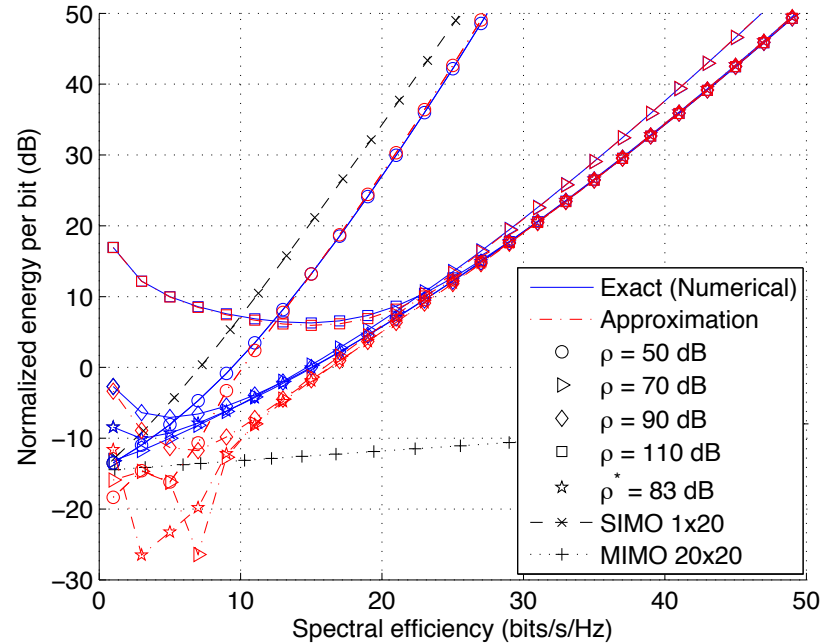

Fig. 4. The EE-SE trade-off with varying Phase I transmit power $\rho$ (H-H scenario, $r=20, \varepsilon=0.5$ ).

\section{A. Impacts of protocol optimization ( $\rho$ and $\varepsilon$ )}

Taking the H-H scenario as an example, Fig. 4 shows the impact of Phase I broadcast power $\rho$ on the EE-SE tradeoff. Both numerical results and theoretical approximations are presented based on (26) and (36), respectively. As expected, the theoretical approximations are shown to be valid in the high SE regime. For comparison purpose, the EE-SE trade-off of $1 \times 20$ SIMO and $20 \times 20$ MIMO systems are presented. The former represents a system where V-MIMO is not deployed. It is used to indicate the performance gains (benefits) of VMIMO. The later represents a system where all mobile users have the same number of antennas as the BS. It indicates the performance upper bound of V-MIMO systems. It is observed that a relatively low broadcasting power (e.g., $\rho=50,70 \mathrm{~dB}$ ) gives unsatisfactory performance in the high SE regime. This is because the maximum number of antennas in a VAA is not achieved. On the contrary, given a relatively high power (e.g., $\rho=90,100 \mathrm{~dB}$ ), the trade-off performance is worsen in the medium SE regime without bringing further improvements into the high SE regime. This is because at high values of $\rho$, Phase I broadcasting capacity becomes interference-limited. Using the heuristic algorithm proposed in Section $\mathrm{V}$ and apply (45) with $k=99 \%$, we are able to calculate $\rho^{*}=83 \mathrm{~dB}$, which is shown to give a satisfactory performance in both the medium and high SE regimes.

Similar to Fig. 4, Fig. 5 shows the impact of time allocation parameter $\varepsilon$ on the EE-SE trade-off in the $\mathrm{H}-\mathrm{H}$ scenario. It is observed that the EE-SE performance improves when $\varepsilon$ increases from 0.1 to 0.7 . However, when $\varepsilon$ further increases from 0.7 to 0.9 , the performance improves slightly in the lower SE regime $(S<20$ bits $/ \mathrm{s} / \mathrm{Hz})$, but quickly gets worse in the higher SE regime $(S>20 \mathrm{bits} / \mathrm{s} / \mathrm{Hz})$. To explain this phenomenon, we note that the essence of V-MIMO is to sacrifice a fraction of time in exchange for higher transmission rates resulting from spatial multiplexing gains. When $\varepsilon$ increases, the useful time fraction increases but the average multiplexing 


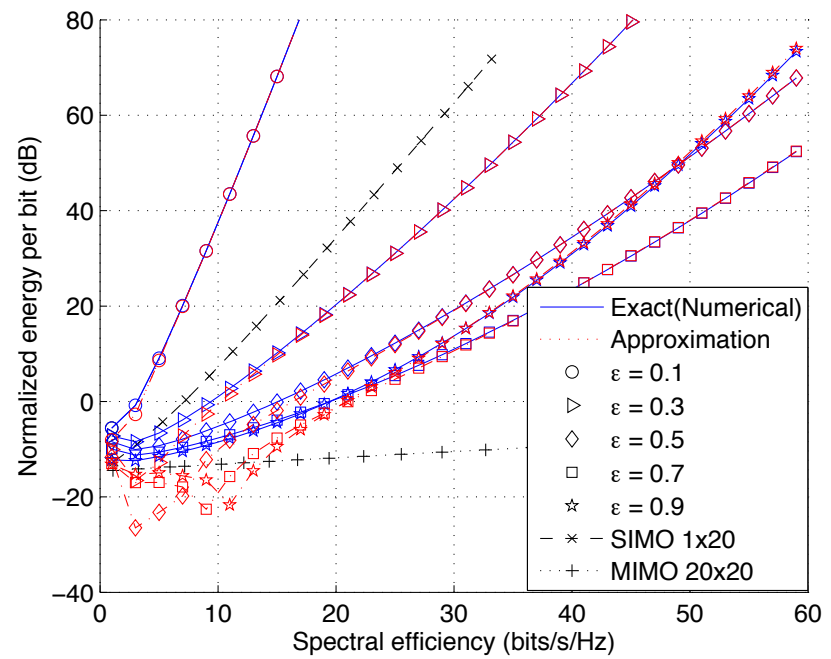

Fig. 5. The EE-SE trade-off with varying time allocation parameter $\varepsilon$ (H-H scenario, $\left.r=20, \rho^{*}=83 \mathrm{~dB}\right)$.

gain decreases. Because the multiplexing gain is particularly useful in the high SE regime, a performance degradation to the high SE regime can be clearly observed when $\varepsilon$ approaches its maximum value 1 . In the extreme case of $\varepsilon=1$, the V-MIMO system reduces to a $1 \times r$ SIMO system with a multiplexing gain of 1 .

As shown in Fig. 5, there does not exist an absolutely optimal $\varepsilon$ that gives the best performance in all SE values. In practice, it is useful to optimize $\varepsilon$ at a given value of SE. This SE value could be the highest SE or the average SE of a system. Taking the LTE-Advanced system for example, the targeted peak SE is $30 \mathrm{bits} / \mathrm{s} / \mathrm{Hz}$. Taking this SE value, in Fig. 6 we show EE as a function of $\varepsilon$ in four scenarios. It is observed that the optimal values of $\varepsilon$ vary from scenario to scenario. Compared with a fixed time allocation scheme of $\varepsilon=0.5$, the optimal $\varepsilon$ gives a EE gain of $10 \mathrm{~dB}, 0 \mathrm{~dB}, 4 \mathrm{~dB}$, and $80 \mathrm{~dB}$ in the H-H, H-L, L-H and L-L scenarios, respectively. We note that the large gain achieved in the L-L scenario is for knowing that V-MIMO is actually harmful and should be reduced to a conventional SIMO system (i.e., $\varepsilon=1$ ).

To further illustrate the usefulness of protocol optimization, in Fig. 7 we compare the EE-SE trade-off curves corresponding to optimized and non-optimized $\rho$ and $\varepsilon$. The optimization is performed at $\mathrm{SE}=30 \mathrm{bits} / \mathrm{s} / \mathrm{Hz}$. In the non-optimized case, we fix $\rho=103 \mathrm{~dB}$ and $\varepsilon=0.5$, which are optimal values for the H-L scenario. It is observed that adaptive optimization of $\rho$ and $\varepsilon$ is particularly useful for the $\mathrm{H}-\mathrm{H}$ and L-L scenarios. A modest improvement is shown for the L-H scenario, mostly because this scenario has the same $\lambda_{v}$ as the H-L scenario. In modern cellular networks, the density of active users $\lambda_{v}$ varies hugely across space and time. Fig.7 clearly demonstrate that in a dynamic cellular network with varying user densities, adaptive optimization of $\rho$ and $\varepsilon$ is indispensable to guarantee that V-MIMO can always operate in a beneficial fashion.

From our analysis in Section IV.A, we know that when SE approaches infinity, the gradient of V-MIMO EE-SE tradeoff curves will eventually become larger than SIMO systems.

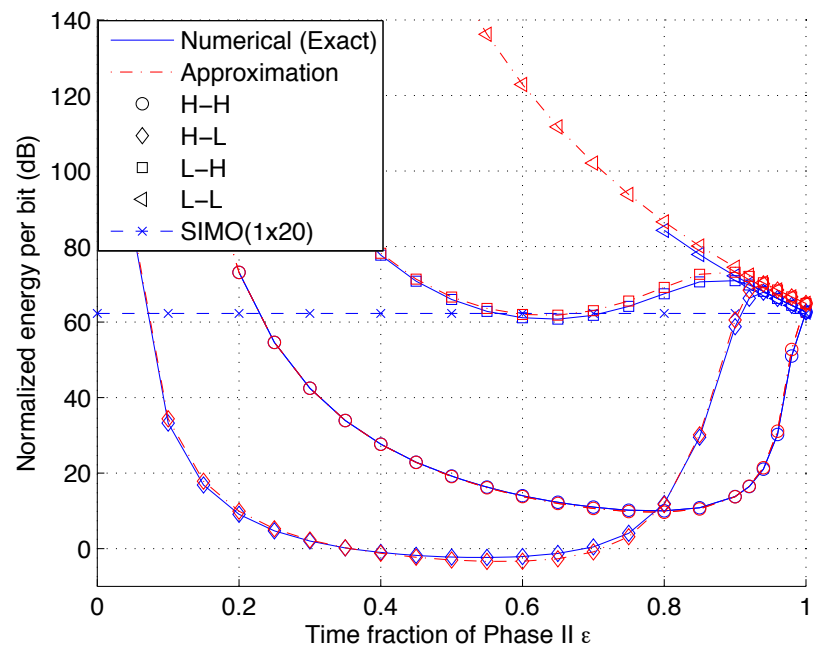

Fig. 6. EE as a function of time allocation parameter $\varepsilon$ in four scenarios $\left(r=20, \mathrm{SE}=30 \mathrm{bit} / \mathrm{s} / \mathrm{Hz}, \rho=\rho^{*}\right)$.

This means that the trade-off curve of any V-MIMO system will eventually surpass the curve of the corresponding SIMO system, therefore V-MIMO scheme is only beneficial within a certain range of SE. In Fig. 8, we show how the gradient of the EE-SE trade-off curve changes with $S$ given different $\varepsilon$ in four scenarios. The gradients are obtained by computing numerically the derivatives of the EE-SE trade-off curves. With increasing SE, the gradients of V-MIMO initially stay lower than the gradient of SIMO, but quickly surpass the SIMO threshold, rise to a peak, and finally decline and converge to the theoretical limit given by (37). Because the gradient can reach much higher values than the ultimate limits, Fig. 8 implies that the benefits of V-MIMO over conventional SIMO systems quickly diminish with increasing $S$.

\section{B. Impacts of scenario characteristics $\left(\lambda_{b}, \lambda_{u}, \lambda_{v}\right.$, and $\left.r\right)$}

Scenario characteristics are practical constraints that cannot be optimized by protocols. Related parameters in our system model include the density parameters $\lambda_{v}, \lambda_{u}, \lambda_{b}$ and the number of BS antennas $r$.

First, let us revisit Figs. 6-8. In all these figures, scenario $\mathrm{H}-\mathrm{L}$ is shown to achieve superior performance, followed by $\mathrm{H}-\mathrm{H}, \mathrm{L}-\mathrm{H}$, and L-L scenarios. It is easy to understand that the $\mathrm{H}-\mathrm{H}$ and $\mathrm{H}-\mathrm{L}$ scenarios outperform L-H and L-L scenarios because the performance of V-MIMO benefits from a higher density of users. Moreover, given the same user density, the H$\mathrm{L}$ scenario outperforms $\mathrm{H}-\mathrm{H}$ because more inactive users are available to form VAAs. But why does L-L fails to outperform $\mathrm{L}-\mathrm{H}$ in a similar fashion? The answer to this lies in (12). Recall our system model in Section II, the bandwidth used in Phase I is on average $\lambda_{v} / \lambda_{b}$ times greater than the bandwidth used in Phase II. This 'Phase I bandwidth gain' can greatly reduce the required received SINR and hence boost the chance for an inactive user to become a VAA participant. In the $\mathrm{L}-\mathrm{L}$ scenario, the bandwidth gain is much lower than L-H, therefore a worse performance is observed. Figs. 6-8 clearly demonstrate that a low frequency reuse factor in Phase I is critical for V-MIMO to be beneficial. 


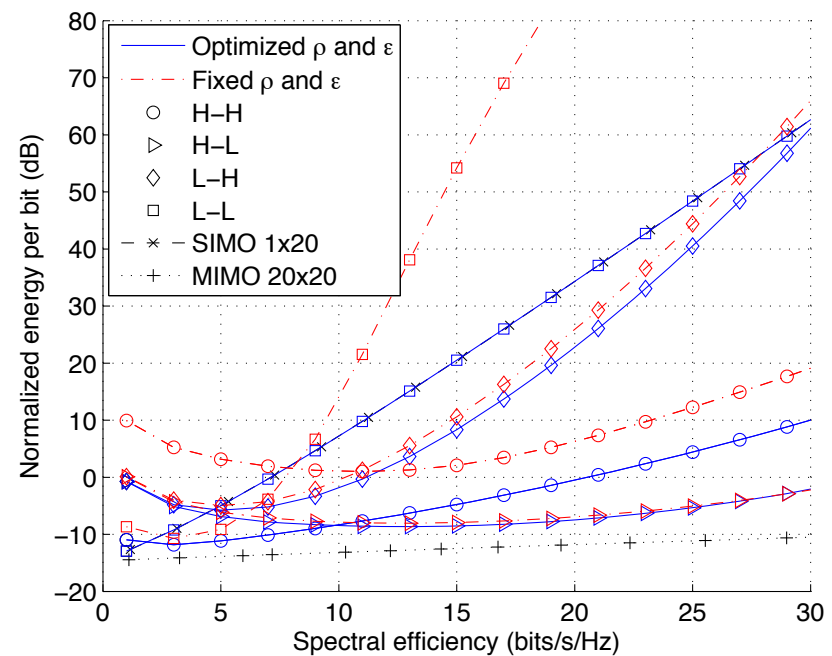

Fig. 7. EE-SE trade-off with and without protocol parameter optimization in four scenarios $(r=20$; In the non-optimized case $\rho=103 \mathrm{~dB}$ and $\varepsilon=0.5$; In the optimized case and for the four scenarios $(\mathrm{H}-\mathrm{H}, \mathrm{H}$ $\mathrm{L}, \mathrm{L}-\mathrm{H}$, and L-L), $\rho^{*}=83 \mathrm{~dB}, 103 \mathrm{~dB}, 103 \mathrm{~dB}$, and $123 \mathrm{~dB}$, respectively, and $\varepsilon^{*}=0.8,0.6,0.6$, and 1 , respectively. Parameter $\varepsilon$ is optimized at $\mathrm{SE}=30 \mathrm{bit} / \mathrm{s} / \mathrm{Hz})$.

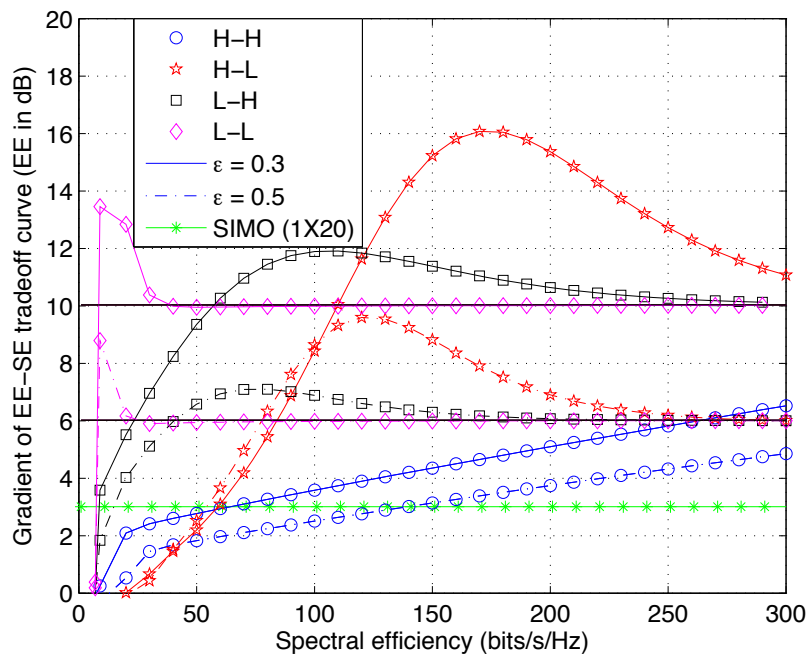

Fig. 8. Gradient of the EE-SE trade-off curve with varying $\varepsilon$ in four scenarios $\left(r=20, \rho=\rho^{*}\right)$.

From above discussions we know that given $\lambda_{u}$ and $\lambda_{b}$, when $\lambda_{v}$ increases, the 'Phase I bandwidth gain' increases but the inactive user density decreases, resulting in conflicting effects on the V-MIMO performance. This inherent tension leads us to a new question: How does the performance of V-MIMO scales up with increasing $\lambda_{v}$ ? Is there an optimal value of $\lambda_{v}$ ? In Fig. 9, we show the EE gain of V-MIMO over SIMO as a function of SE with varying $\lambda_{v}$. Numerical methods in (26) and theoretical approximations in (36) are used to compute the EE in the medium SE regime and high SE regime, respectively. With increasing $\lambda_{v} / \lambda_{b}$, we observe a general trend that the EE gain increases initially before eventually diminishes. Interestingly, the changes in performance do not

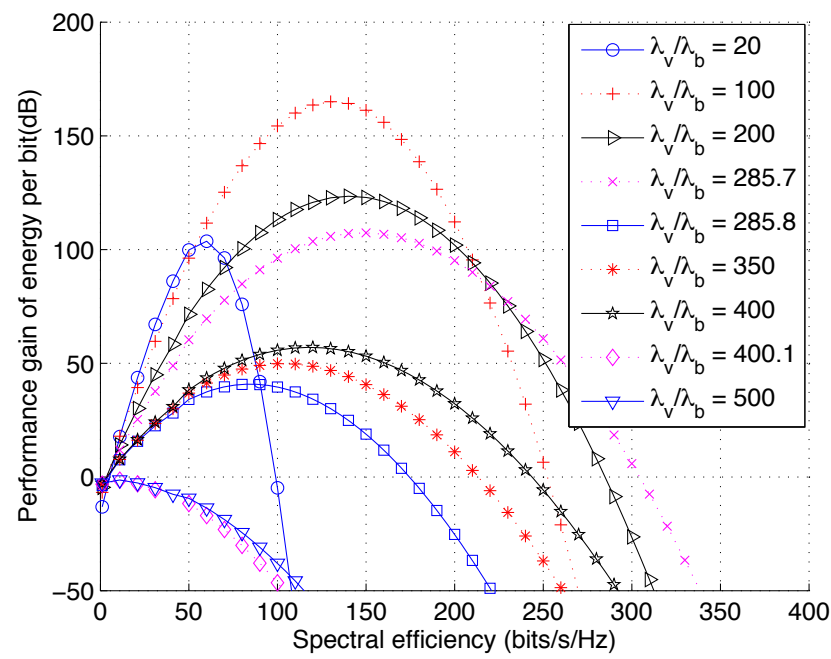

Fig. 9. EE gain as a function of SE with varying $\lambda_{v} / \lambda_{b}\left(\lambda_{u} / \lambda_{b}=1000\right.$, $r=20, \rho=\rho^{*}, \varepsilon=0.5$ ).

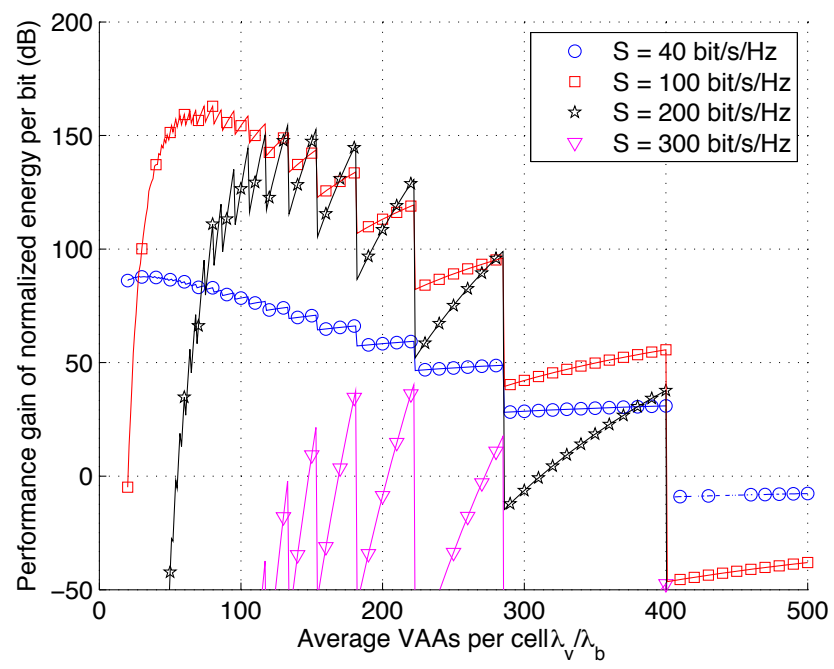

Fig. 10. EE gain as a function of $\lambda_{v} / \lambda_{b}$ with varying SE $\left(\lambda_{u} / \lambda_{b}=1000, r=20, \rho=\rho^{*}, \varepsilon=0.5\right)$.

follow a smooth transition as one would normally expect, but exhibits chaotic behaviors. For example, at $\lambda_{v} / \lambda_{b}=400$, VMIMO is shown to achieve a considerable EE gain of up to $55 \mathrm{~dB}$ within a wide range of SE values ranging from several bits $/ \mathrm{s} / \mathrm{Hz}$ to about $250 \mathrm{bits} / \mathrm{s} / \mathrm{Hz}$. After a tiny increase on $\lambda_{v} / \lambda_{b}$ to 400.1 , all the gains disappear and V-MIMO suddenly shows no benefits compared with a SIMO system. A similar phenomenon is observed when $\lambda_{v} / \lambda_{b}$ increases from 285.7 to 285.8.

To better understand the chaotic behavior of V-MIMO, in Fig. 10 we show the EE gain as a function of $\lambda_{v} / \lambda_{b}$ at different values of SE. The curve of $\mathrm{SE}=40 \mathrm{bits} / \mathrm{s} / \mathrm{Hz}$ is computed using numerical methods based on (26), while the other curves are computed using theoretical approximation based on (36). A 'saw-like' shape is observed for all curves. It appears that the entire range of continuous-valued $\lambda_{v} / \lambda_{b}$ can be divided into many intervals. Within each interval, the EE gain raises with 


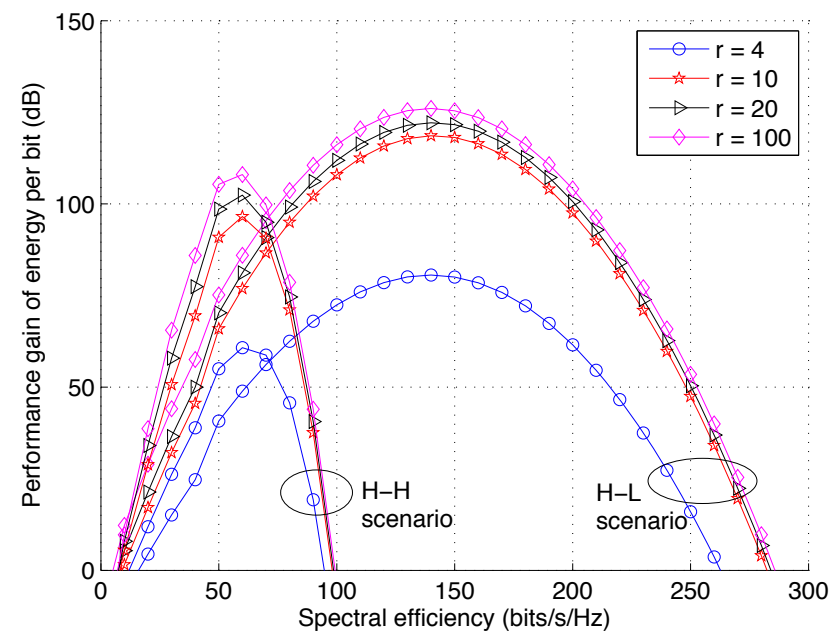

Fig. 11. EE gain as a function of SE with varying $r$ in the $\mathrm{H}-\mathrm{H}$ and H-L scenarios $\left(\rho=\rho^{*}, \varepsilon=0.5\right)$.

$\lambda_{v} / \lambda_{b}$. But at the end of each interval, the EE gain takes a sudden decline. To our best knowledge, this is the first time that such a chaotic behavior is reported for V-MIMO systems. A detailed investigation into this phenomenon is out of the scope of this paper and is left for future work.

Let's now consider the impact of $r$ on the performance of V-MIMO. In Fig. 11, we show the EE gain as a function of SE with varying $r$ in the H-H and H-L scenarios. It is observed that the EE gains increase with increasing $r$. However, the increase in EE gain is not significant once $r$ is greater than 10 . The practical implication is that deploying very large antenna arrays at the BS is not particularly useful to harness more gains from V-MIMO.

\section{Low SE regime and approximation accuracy}

Our previous discussions have focused entirely on medium and high SE regimes. For completeness of our discussion, in Fig. 12 we show the EE-SE trade-off curve at the low SE regime, where the $\mathrm{SE}$ values are shown in $\mathrm{dB}$ scale. We can see that EE increases with decreasing SE, which is because a fixed power $\rho^{*}$ has been consumed in Phase I. In addition, the trade-off performance degrades with increasing $\varepsilon$.

Finally, we discuss briefly the approximation accuracy of (36) and (38). In the high SE regime, the approximation error mainly comes from (31). Only when $\tilde{\chi}>40 \mathrm{~dB}$, the approximation error of (31) becomes less than one percent. Correspondingly, the approximation in (36) is deemed acceptable when $\mathrm{SE}>4 \min (\bar{t}, r)$ bits/s/Hz, where $\bar{t}$ is the average VAA size and $r$ is the number of antennas at the BS. As we can observe in Figs. 4 and 5, the approximation is very good for SE $>40$ bits $/ \mathrm{s} / \mathrm{Hz}$ in our system setting. Similarly, in the low SE regime, the approximation in (38) is deemed acceptable when $\mathrm{SE}<0.01 \sqrt{r / \varepsilon}$ bits/s/Hz, where $\varepsilon$ is the time fraction of Phase II. As we can observe in Fig. 12, theoretical approximation is shown to agree well with numerical results for $\mathrm{SE}<0.1 \mathrm{bits} / \mathrm{s} / \mathrm{Hz}$.

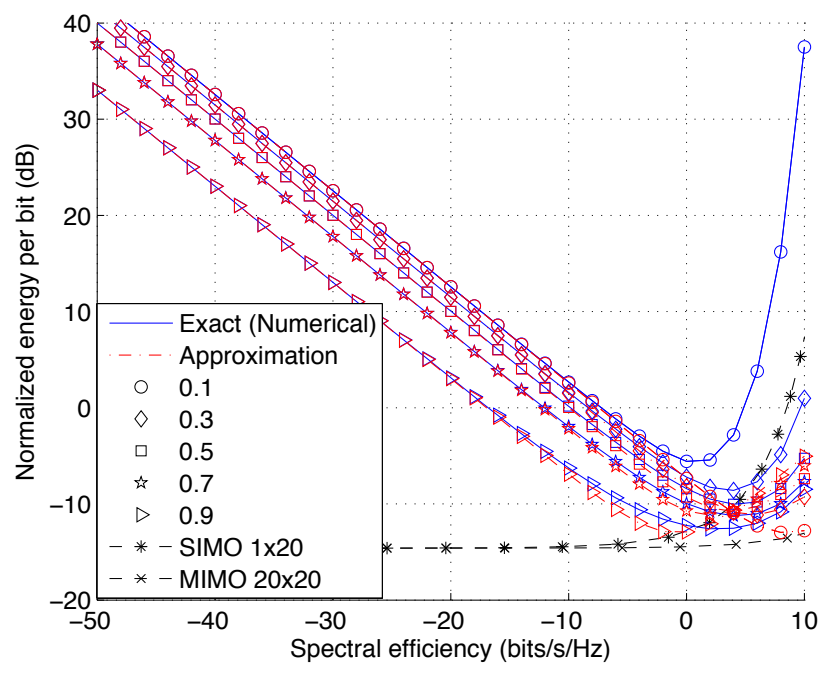

Fig. 12. The EE-SE trade-off at the low SE regime with varying time allocation parameter $\varepsilon\left(\mathrm{H}-\mathrm{H}\right.$ scenario, $r=20, \rho^{*}=83 \mathrm{~dB}$, the $\mathrm{SE}$ values are shown in the $\mathrm{dB}$ scale).

\section{CONCLUSIONS}

This paper has studied the EE-SE trade-off of a large-scale V-MIMO system with decode-and-forward type protocols. A general framework has been established to numerically calculate EE as a function of SE. Closed-form approximations of the trade-off function have been derived for both the high SE regime and low SE regime and shown to be accurate for $\mathrm{SE}>40 \mathrm{bits} / \mathrm{s} / \mathrm{Hz}$ and $\mathrm{SE}<0.1 \mathrm{bits} / \mathrm{s} / \mathrm{Hz}$, respectively. The impacts of power allocation, time fraction allocation, user density, VAA density, BS antenna number, and frequency reuse factor on the EE-SE trade-off have been thoroughly investigated. A heuristic power control algorithm has been provided to simplify the process of trade-off optimization. It has been found that the V-MIMO system exhibits a chaotic behavior uncommon to conventional communication systems. We conclude that from a system perspective, the key of VMIMO system design and optimization lies on low frequency reuse factors, adaptive resource allocation, and VAA density control.

\section{REFERENCES}

[1] M. Dohler, J. Dominguez, and H. Aghvami, "Link capacity analysis for virtual antenna arrays," Proc. IEEE VTC-Fall'02, Vancouver, Canada, Sept. 2002, pp. 440-443.

[2] M. Dohler, A. Gkelias, and H. Aghvami, "Resource allocation for FDMA-based regenerative multi-hop links," IEEE Trans. Wireless Commun., vol. 3, no. 6, pp. 1989-993, Nov. 2004.

[3] M. Dohler, A. Gkelisa, and H. Aghvami, "A resource allocation strategy for distributed MIMO multi-hop communication systems," IEEE Commun. Lett., vol. 8, no. 2, pp. 99-101, Feb. 2004.

[4] Y. Zhou and T.S. Ng, "Performance analysis on MIMO-OFCDM systems with multi-code transmission", IEEE Trans. Wireless Commun., vol. 8, no. 9, pp. 4426-4433, Sept. 2009.

[5] Y. Zhou and T.S. Ng, "MIMO-OFCDM systems with joint iterative detection and optimal power allocation", IEEE Trans. Wireless Commun., vol. 7, no. 12, pp. 5504-5516, Dec. 2008.

[6] C.-X. Wang, X. Hong, X. Ge, X. Cheng, G. Zhang, and J. S. Thompson, "Cooperative MIMO channel models: a survey," IEEE Commun. Mag., vol. 48, no. 2, pp. 80-87, Feb. 2010. 
[7] X. Cheng, C.-X. Wang, H. Wang, X. Gao, X.-H. You, D. Yuan, B. Ai, Q. Huo, L. Song, and B. Jiao, "Cooperative MIMO channel modeling and multi-link spatial correlation properties," IEEE J. Sel. Areas Commun., vol. 30, no. 2, pp. 388-396, Feb. 2012.

[8] C.-X. Wang, X. Hong, H.-H. Chen, and J. Thompson, "On capacity of cognitive radio networks with average interference power constraints," IEEE Trans. Wireless Commun., vol. 8, no. 4, pp. 1620-1625, Apr. 2009.

[9] X. Hong, C.-X. Wang, M. Uysal, X. Ge, and S. Ouyang, "Capacity of hybrid cognitive radio networks with distributed VAAs", IEEE Trans. Veh. Technol., vol. 59, no. 7, pp. 3510-3523, Sep. 2010.

[10] P. Zhang, F. Wang, L. Tu, K. Li, S. Diouba, and I. Khider, "Opportunistic virtual antenna array with optimal relay mobile terminals selection," Proc. 4th Int. Conf. WiCOM, Oct. 2008, pp. 1-4.

[11] L. Wang and L. Hanzo, "The resource-optimized differentially modulated hybrid $\mathrm{AF} / \mathrm{DF}$ cooperative cellular uplink using multiple-symbol differential sphere detection," IEEE Signal Process. Lett., vol. 16, no. 11, pp. 965-968, Nov. 2009.

[12] X. Ge, K. Huang, C.-X. Wang, X. Hong, and X. Yang, "Capacity analysis of a multi-cell multi-antenna cooperative cellular network with co-channel interference," IEEE Trans. Wireless Commun., vol. 10, no. 10, pp.3298-3309, Oct. 2011.

[13] S. Cui, A. J. Goldsmith, and A. Bahai, "Energy-efficiency of MIMO and cooperative MIMO techniques in sensor networks," IEEE J. Sel. Areas Commun., vol. 22, no. 6, pp. 1089-1098, Aug. 2004.

[14] J. Liang and Q. Liang, "Channel selection in virtual MIMO wireless sensor networks," IEEE Trans. Veh. Technol., vol. 58, no. 5, pp. 22492257, June 2009.

[15] S. Jayaweera, "V-BLAST virtual MIMO for distributed wireless sensor networks," IEEE Trans. Commun., vol. 55, no. 10, pp. 1867-1872, Oct. 2007.

[16] H. Gharavi, B. Hu, and N. Wu, "A design framework for high-density wireless ad-hoc networks achieving cooperative diversity," Proc. IEEE ICC, Cape Town, South Africa, May 2010, pp. 1-5.

[17] C. Buratti and A. Zanella, "Multihop virtual MIMO systems with channel reuse in a Poisson field of nodes," IEEE Trans. Veh. Technol., vol. 60, no. 5, pp. 2060-2069, June 2011.

[18] A. del Coso, U. Spagnolini, and C. Ibars, "Cooperative distributed MIMO channels in wireless sensor networks," IEEE J. Sel. Areas Commun., vol. 25, no. 2, pp. 402-414, Feb. 2007.

[19] X. Li, "Energy efficient wireless sensor networks with transmission diversity," Electron. Lett., vol. 39, no. 24, pp. 1753-1755, Nov. 2003.

[20] W. Deng and X. Gao, "Three-hop cooperative diversity system and symbol error rate analysis," Proc. Int. Symp. ISPACS, Xiamen, China, Nov. 2007, pp. 606-609.

[21] L. Tian, Y. Zhou, Y. Zhang, G. Sun and J. L. Shi, "Resource allocation for multicast services in distributed antenna systems with QoS guarantees", IET Commun., vol. 6, no. 3, pp. 264-271, March 2012.

[22] H. Chen, A. B. Gershman, and S. Shahbazpanahi, "Filter-and-forward distributed beamforming in relay networks with frequency selective fading," IEEE Trans. Signal Processing, vol. 58, no. 3, Mar. 2010, pp. 1251-1262.

[23] Z. Wang, F. Yuan, L. Xiang, and L. Zheng, "Distributed beamforming design on amplify and forward two-way relaying channels," Proc. 2nd Intl. Conf. ICDMA, Zhangjiajie, China, Apr. 2011, pp. 433-437.

[24] M. D. Renzo, F. Graziosi, and F. Santucci, "On the performance of CSI assisted cooperative communications over generalized fading channels," Proc. IEEE ICC, Beijing, China, May 2008, pp. 1001-1007.

[25] Y. Yuan, Z. He, and M. Chen, "Virtual MIMO-based cross-layer design for wireless sensor networks," IEEE Trans. Veh. Technol., vol. 55, no. 3, pp. 856-864, May 2006.

[26] S. Simoens, O. M. Medina, J. Vidal, and A. D. Coso, "Compress-andforward cooperative MIMO Relaying with full channel state information," IEEE Trans. Signal Process., vol. 58, no. 2, pp. 781-791, Feb. 2010.

[27] J. Jiang, J. S. Thompson, H. Sun, and P. M. Grant, "Performance assessment of virtual multiple-input multiple-output systems with compress-and-forward cooperation," IET Commun., vol. 6, no. 11, Nov. 2012, pp. 1456-1465.

[28] Y. Lang, D. Wubben, C. Bockelmann, and K.-D. Kammeyer, "A closed power allocation solution for outage restricted distributed MIMO multihop networks," Proc. 6th Intl. Symposium WiOpt, Berlin, Germany, Apr. 2008, pp. 65-70.

[29] L. Xiang, X. Ge, C.-X. Wang, F. Y. Li, and F. Reichert, "Energy efficiency evaluation of cellular networks based on spatial distributions of traffic load and power consumption," IEEE Trans. Wireless Commun., vol. 12, no. 3, pp. 961-973, Mar. 2013.
[30] S. Verdu, "Spectral efficiency in the wideband regime," IEEE Trans. Inf. Theory, vol. 48, no. 6, pp. 1319-1343, June 2002.

[31] F. Haider, C.-X. Wang, X. Hong, H. Haas, D. Yuan, and E. Hepsaydir, "Spectral-energy efficiency tradeoff in cognitive radio networks with peak interference power constraints," Proc. IEEE ICCT'11, Jinan, China, Sept. 2011.

[32] A. Lozano, A. M. Tulino, and S. Verdu, "Multiple-antenna capacity in the low-power regime," IEEE Trans. Inf. Theory, vol. 49, no. 10, pp. 2527-2544, Oct. 2003.

[33] F. Heliot, M. A. Imran, and R. Tafazolli, "On the energy efficiencyspectral efficiency trade-off over the MIMO Rayleigh fading channel," IEEE Trans. Commun., vol. 60, no. 5, pp. 1345-1356, May 2012.

[34] G. Caire, G. Taricco, and E. Biglieri, "Suboptimality of TDMA in the low-power regime," IEEE Trans. Inf. Theory, vol. 50, no. 4, pp. 608-620, Apr. 2004.

[35] C.-L. Chen, W. E. Stark, and S.-G. Chen, "Energy-bandwidth efficiency trade-off in MIMO multi-hop wireless networks," IEEE J. Sel. Areas Commun., vol. 29, no. 8, pp. 1537-1546, Sept. 2011.

[36] Y. Yao, X. Cai, and G. B. Giannakis, "On energy efficiency and optimum resource allocation of relay transmissions in the low-power regime," IEEE Trans. Wireless Commun., vol. 4, no. 6, pp. 2917-2927, Nov. 2005.

[37] O. Somekh, B. M. Zaidel, and S. S hamai, "Sum rate characterization of joint multiple cell-site processing," IEEE Trans. Inf. Theory, vol. 53, no. 12, pp. 4473-4497, Dec. 2007.

[38] O. Simeone, O. Somekh, Y. Bar-Ness, and U. Spagnolini, "Throughput of low-lower cellular systems with collaborative base stations and relaying," IEEE Trans. Inf. Theory, vol. 54, no. 1, pp. 459-467, Jan. 2008.

[39] F. Haider, C.-X. Wang, H. Haas, D. Yuan, H. Wang, X. Gao, X.-H. You, and E. Hepsaydir, "Spectral-energy efficiency tradeoff for mobile femtocell networks with resource partitioning schemes," Proc. IEEE ICCT11, Jinan, China, Sept. 2011, pp. 1-5.

[40] S. Shamai and S. Verdu, "The impact of frequency-flat fading on the spectral effeciency of CDMA," IEEE Trans. Inf. Theory, vol. 47, no. 4, pp. 1302-1327, May 2001.

[41] A. Lozano, A. Tulino, and S. Verdu, "High-SNR power offset in multiantenna communication," IEEE Trans. Inf. Theory, vol. 51, no. 12, pp. 4134-4151, Dec. 2005

[42] N. Jindal, "High SNR analysis of MIMO broadcast channels," Proc. IEEE ISIT, Adelaide, Australia, Sept. 2005, pp. 1-5.

[43] D. Stoyan, W. S. Kendall, and J. Mecke, Stochastic Geometry and Its Applications, 2nd Edition, Wiley, 2008.

[44] J. G. Andrews, F. Bacccelli, and R. K. Ganti, "A tractable approach to coverage and rate in cellular networks," IEEE Trans. Commun., vol. 59, no. 11 , pp. 3122-3134, Nov. 2011

[45] C.-X. Wang, X. Hong, H. Wu, and W. Xu, "Spatial temporal correlation properties of the 3GPP spatial channel model and the Kronecker MIMO channel model", EURASIP J. Wireless Commun. and Networking, vol. 2007, Article ID 39871, 9 pages, 2007.

[46] S. M. Yu and S.-L. Kim, "Downlink capacity and base station density in cellular networks," IEEE Trans. Veh. Technol., submitted.

[47] E. Biglieri and G. Taricco, Transmission and Reception with Multiple Antennas: Theoretical Foundations, Now Publishers Inc., 2004.

[48] D. Tse and P. Viswanath, Fundamentals of wireless communication, Cambridge Unviersity Press, 2005

[49] K. Yang, S. Ou, K. Guild, and H.H. Chen. "Convergence of Ethernet PON and IEEE 802.16 broadband access networks and its QoS-aware dynamic bandwidth allocation scheme", IEEE J. Sel. Areas Commun., vol. 27, no. 2, pp. 101-116, Feb. 2009.

[50] G. Zhang, K. Yang, P. Liu, E. Ding, and Y. Zhong, Joint channel bandwidth and power allocation game for selfish cooperative communication networks, IEEE Trans. Veh. Technol., vol. 61, no. 9, pp. 4142-4156, Nov. 2012. 


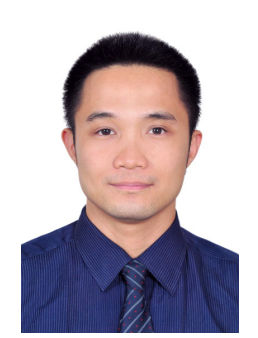

Xuemin Hong (S'05-M'12) received a PhD degree from Heriot-Watt University, UK, in 2008. Since 2011, he has been an associate professor at $\mathrm{Xi}$ amen University, China. From 2009 to 2011, he was a post-doc research fellow at the University of Waterloo, Canada and Heriot-Watt University, UK.

Dr Hong's research interests include MIMO and cooperative systems, wireless channel modelling, cognitive radio networks, and wireless ad hoc networking. He has published more than 20 technical papers in major international journals and conferences and 1 book chapter in the area of wireless communications. He served as a member of the Technical Program Committee for a number of international conferences.

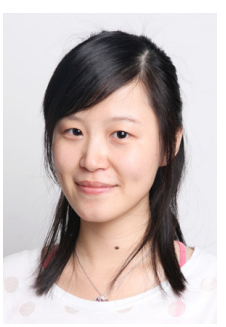

Jie Yu received her B.Eng. degree from Xiamen University of Technology, Xiamen, Fujian, China, in 2010. She is currently a post-graduate student at Xiamen University, Xiamen, Fujian, China. Her research interests include cooperative MIMO systems and cognitive radio networks.

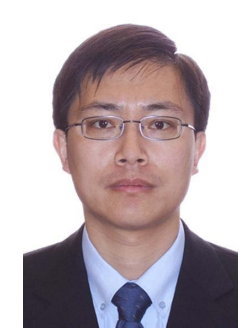

Cheng-Xiang Wang (S'01-M'05-SM'08) received the B.Sc. and M.Eng. degrees in communication and information systems from Shandong University, Shandong, China, in 1997 and 2000, respectively, and the Ph.D. degree in wireless communications from Aalborg University, Aalborg, Denmark, in 2004.

Since 2005, he has been with Heriot-Watt University, Edinburgh, U.K., first as a Lecturer, then as a Reader in 2009, and as a Professor in 2011. $\mathrm{He}$ is also an Honorary Fellow of the University of Edinburgh and a Chair/Guest Professor with Shandong University; Huazhong University of Science and Technology, Wuhan, Hubei, China; and Southeast University, Nanjing, China. From 2001 to 2005, he was a Research Fellow with the University of Agder, Grimstad, Norway. In 2004, he was a Visiting Researcher with Siemens AG-Mobile Phones, Munich, Germany. From 2000 to 2001, he was a Research Assistant with the Technical University of Hamburg-Harburg, Hamburg, Germany. He is the Editor of one book. He has published one book chapter and over 180 papers in refereed journals and conference proceedings. His research interests include wireless channel modeling and simulation, green communications, cognitive radio networks, vehicular communication networks, large multiple-input multipleoutput (MIMO), cooperative MIMO, and Beyond-Fourth-Generation wireless communications.

Prof. Wang is a Fellow of the Institution of Engineering and Technology and the HEA and a member of the Engineering and Physical Research Council Peer Review College. He has served as an editor for eight international journals, including the IEEE TRANSACTIONS ON VEHICULAR TECHNOLOGY (since 2011) and the IEEE TRANSACTIONS ON WIRELESS COMMUNICATIONS (2007-2009). He was the lead Guest Editor for the IEEE JOURNAL ON SELECTED AREAS IN COMMUNICATIONS Special Issue on Vehicular Communications and Networks. He has served as a Technical Program Committee (TPC) Member, TPC Chair, and General Chair for more than 70 international conferences. He received the Best Paper Awards at the 2010 IEEE Global Telecommunications Conference, the 13th IEEE International Conference on Communication Technology in 2011, and the 12th International Conference on ITS Telecommunications in 2012.

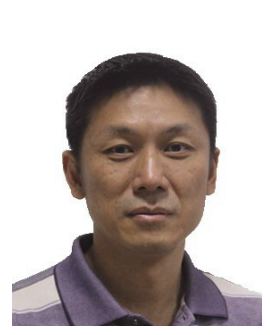

Jianghong Shi received his PhD from Xiamen University, China, in 2002 . He is currently a professor in the School of Information Science and Technology, Xiamen University. $\mathrm{He}$ is also the director of the West Straits Communications Engineering Center, Fujian Province, China. His research interests include wireless communication networks and satellite navigation.

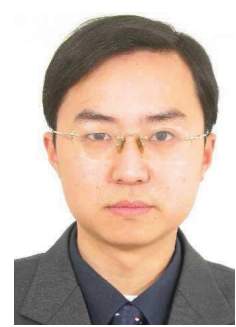

Xiaohu Ge (M'09-SM'11) is currently a Professor with the Department of Electronics and Information Engineering at Huazhong University of Science and Technology (HUST), China. He received his $\mathrm{PhD}$ degree in Communication and Information Engineering from HUST in 2003. He has been working at HUST since Nov. 2005. Prior to that, he worked as a researcher at Ajou University (Korea) and Politecnico Di Torino (Italy) from Jan. 2004 to Oct. 2005. He was a visiting researcher at Heriot-Watt University, Edinburgh, UK from June to August 2010. His research interests are in the area of mobile communications, traffic modeling in wireless networks, green communications, and interference modeling in wireless communications. He has published about 60 papers in refereed journals and conference proceedings and has been granted about 15 patents in China. He received the Best Paper Award from IEEE Globecom 2010. He is leading several projects funded by NSFC, China MOST, and industries. He is taking part in several international joint projects, such as the RCUK funded UK-China Science Bridges: R\&D on (B)4G Wireless Mobile Communications and the EU FP7 funded project: Security, Services, Networking and Performance of Next Generation IP-based Multimedia Wireless Networks.

Dr. Ge is currently serving as an Associate Editor for International Journal of Communication Systems (John Wiley \& Sons), Journal of Internet Technology and KSII Transactions on Internet and Information Systems. Since 2005 , he has been actively involved in the organisation of more than 10 international conferences, such as Executive Chair of IEEE GreenCom 2013 and Co-Chair of workshop of Green Communication of Cellular Networks at IEEE GreenCom 2010. He is a Senior Member of the IEEE, a Senior member of the Chinese Institute of Electronics, a Senior member of the China Institute of Communications, and a member of the NSFC and China MOST Peer Review College. 